\section{The function of spatial frequency analysis: Test of a proposal}

\author{
ANGUS R. H. GELLATLY \\ University of Keele, Keele, Staffs, England
}

Ever since Campbell and Robson (1968) proposed that the visual system might comprise three or more spatial frequency channels, visual scientists have been faced by two major types of question: (1) Is the proposal correct? Does the visual system in fact perform a rough frequency analysis? (2) If so, why? What would having three or more descriptions of a scene buy for the system? Over the years, a great deal of evidence has accumulated in connection with the first type of question, much of it seeming to favor affirmative answers. On the other hand, little progress has been made in even conceiving of why a frequency analysis might be functional, much less in obtaining data to support or refute particular conceptions. This paper reports research concerning the second type of question, which is related to a specific answer that has been proposed to it.

Although few attempts have been made to provide a functional explanation for the evolution of the putative frequency analyzers, one suggestion that has been proposed relates them to attention. Julesz (1980; Julesz \& Schumer, 1981) argued that if the human contrast sensitivity function reflects the envelope of the sensitivities of three broadly tuned channels (high, medium, and low frequency), these fundamental channels might underlie our ability to widen and narrow at will our field of attention. For instance, when taking in the overall layout of a scene, such as in a lecture theater, we would be employing a low-frequency description; in focusing on some medium-sized object, such as the lecturer, we would use a medium-frequency description; and scrutiny of some small feature ("Look at that tie!") would result from high-frequency analysis. The three channels would allow for something perceptually analogous to a zoom lens, enabling the observer to differentially deploy attention over the visual field while remaining stationary. In support of this hypothesis, Julesz and Schumer (1981) cite the findings of Frome and her colleagues (Frome, Levinson, Danielson, \& Clavadetscher, 1979; Levinson \& Frome, 1979). These investigators showed that although adaptation to a particular grating led to an upward shift in perceived frequency of test gratings of higher frequency than that of the adapting grating (Blakemore \& Sutton, 1969), it paradoxically resulted also in a

The author's mailing address is: Department of Psychology, University of Keele, Keele, Staffs. STS SBG, England. perceived widening of single test bars or the bars of a test grating. That is, there appeared to be both more bars per degree in a test grating at the same time that the bars themselves were perceived to be wider. Levinson and Frome offered an explanation of the paradox in terms of a relation between attention and frequency analysis similar to that proposed by Julesz. Specifically, they suggested that for focused attention a window function might be imposed on the observer's visual system such that spatial frequency analysis would be restricted to a limited region of the visual field. When judging the apparent frequency of a grating attention would be deployed over a broad field and the system would respond to the grating frequency, but when judging the width of individual bars attention would be restricted to a single bar at a time and the effective spectrum would be of that bar alone.

It should be noted that, although similar, the proposals of Julesz and of Frome are not identical. Both suppose that the effective visual field can be narrowed or widened at will, a process of attention. But whereas for Julesz this attentional "zoom" capability is a product of spatial frequency analysis, according to the Levinson and Frome hypothesis the analysis takes place only after the extent of the effective (attended) field has been determined. That is, Julesz identifies frequency analysis as a mechanism of visual attention, whereas Levinson and Frome consider frequency analysis and attention to be two separate, but related, processes. As it happens, for the present paper the distinction does not prove to be of importance, and for the moment we will concentrate on the proposal of Julesz (1980). This is the simpler of the two, and in postulating an identity between frequency analysis and visual attention, it allows for certain predictions that can be empirically examined. Subsequent discussion will additionally demonstrate that tests of those predictions also have bearing on the Levinson and Frome hypothesis.

Let us examine what the consequences are of assuming that there exist three spatially tuned channels within the visual system, their output being the basis upon which attention to different levels of detail is possible. What empirical outcomes follow from assumption of such a theory? One general prediction must be that when information at different frequencies is available in the same focal plane the observer ought to be able to attend selectively to some of it and ignore the rest. That is, it should be possible to attend to either high, medium, or low frequencies at will. The majority of studies of the frequency sensitivity of the visual system have relied on single grating stimuli in which only one fundamental frequency is present. If, however, composite gratings that con- 
tain two fundamental frequencies are devised (Figure 1 and Figure 2), then, according to the attentional theory of frequency analysis, it should be possible to attend selectively to one or the other, provided only that they are sufficiently separated in the frequency domain to produce distinguishable outputs from the frequency-sensitive channels. The question then arises of how we are to know when attention is focused on the high-frequency rather than the low-frequency component, or vice versa. What perceptual consequences can be made contingent upon attention to one rather than to the other? A possibility that fairly readily suggests itself involves the use of frequency-contingent color aftereffects. Harris (1970) showed that after adaptation to, for instance, a red and black high-frequency grating alternating with a green and black low-frequency grating, subsequently viewed black and white gratings appeared to be tinged with green if they were high frequency and with pink if they were low frequency. What, we can ask, would happen if, following such adaptation, test gratings such as those in Figures 1 and 2 were employed? If the Julesz theory is correct, then subjects ought to be able to tune in attentionally to either of the fundamental frequencies (provided these have been suitably chosen) and therefore to determine selectively which color of aftereffect to perceive. The remainder of this paper describes tests of this hypothesis that have been carried out.

In general outline, the experiment is a simple one to set up, the only real problem being the choice of appropriate frequencies for adapting and test gratings. Because subjects are to be asked to distinguish

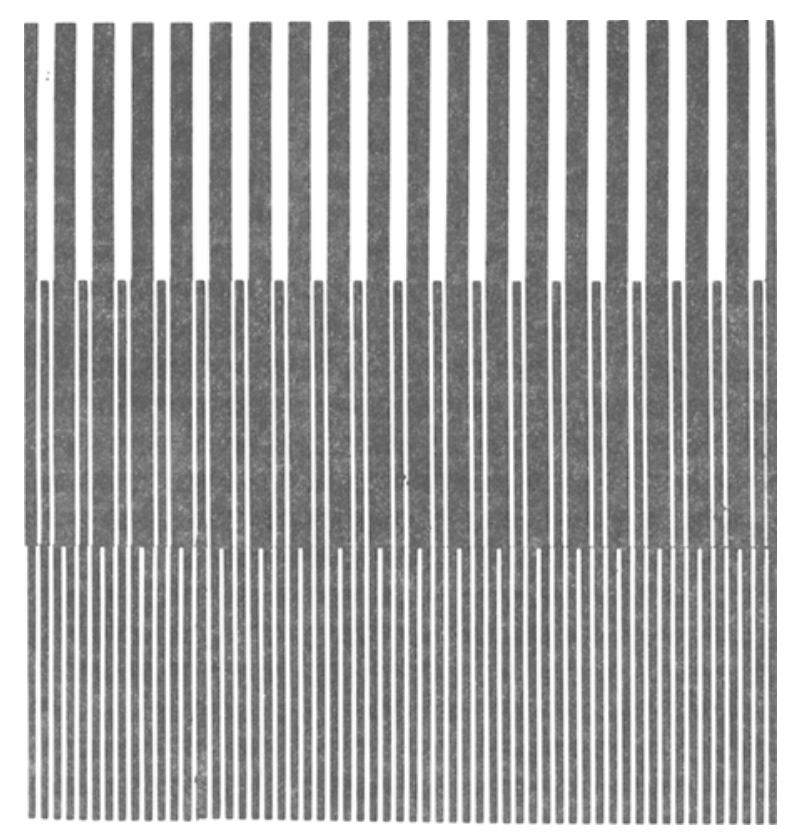

Figure 1

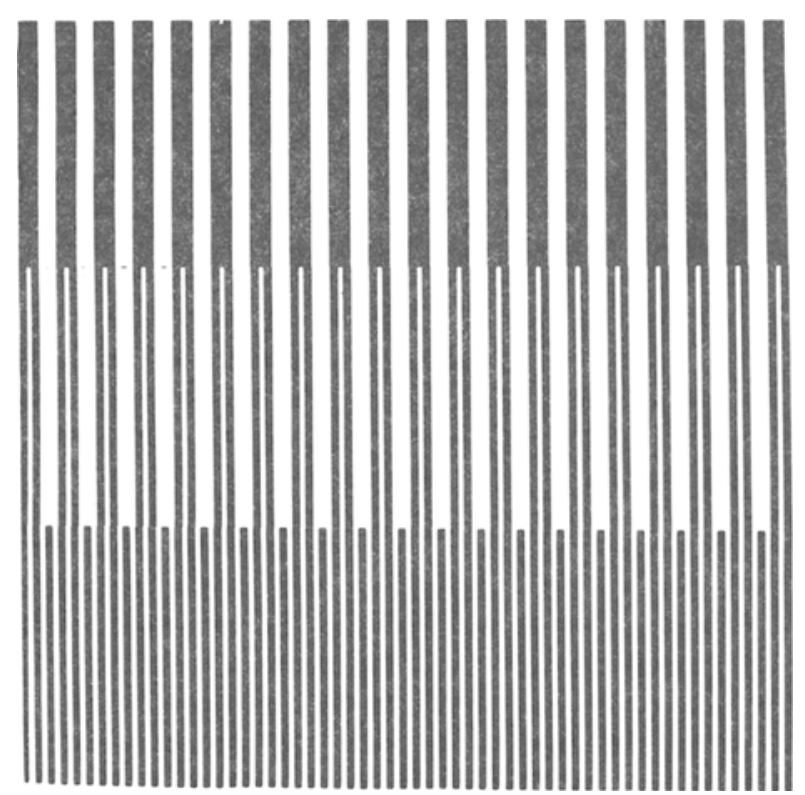

Figure 2

between different colors, it is desirable that the aftereffects be as strong as possible in order to facilitate discrimination. Since the strength of frequencycontingent color aftereffects depends both on the absolute frequencies of the adapting gratings and on the separation between them (Stromeyer, 1978), consideration must be given to both these factors. With square-wave gratings, aftereffects are obtainable over a range of at least 0.5 to 20 cycles/deg, but they are strongest near 5 cycles/deg (Stromeyer, 1978). Different colors may be obtained with gratings that differ by only 1 octave in spatial frequency (Lovegrove \& Over, 1972). From the point of view of obtaining strong colors, a minimal separation in frequency was desirable in the present investigation, since then both gratings could be relatively close to the optimal 5 cycles/deg. On the other hand, by the nature of the gratings in Figures 1 and 2, a minimum 3:1 ratio of stripe widths is necessary, and, in any event, stronger colors might well be obtained with a separation greater than 1 octave. In addition, Julesz (1980) envisioned 2-octave-wide critical bands for his three frequency channels. If selective perception of aftereffects failed with a $3: 1$ ratio, it could be argued that such a result was due to insufficient separation, the frequencies of both gratings falling within the same critical band. Accordingly, adaptation and test gratings were employed in which the ratio of stripe widths varied from $3: 1$ to $5: 1$ to $7: 1$ in different studies, with the actual frequencies centered always around 5 cycles/deg. In other respects, the general procedure was constant in all cases.

A total of 38 subjects participated in three studies. The subjects were tested individually or in groups. 
The adaptation gratings were always red and black and green and black (Wratten filters) and were projected onto a screen. The combination of color with higher and lower frequency gratings was counterbalanced across subjects for each study. During adaptation, the subjects were exposed to alternating 20-sec exposures of the two gratings, with 2-sec intervals between exposures. Adaptation periods were never less than $20 \mathrm{~min}$ and reached $\mathbf{4 0} \mathrm{min}$ for some subjects in the case of the $7: 1$ gratings. Following adaptation, there was a pause of 1 or 2 min before the subjects inspected high-contrast glossy prints of test patterns similar to those in Figures 1 and 2. The frequencies of the test gratings were of course matched to those used for adaptation. The subjects were told that they might be able to see colors on the black and white gratings and that they were free to move about and vary their distance from the test patterns while looking for these colors; they were also told that colors were not invariably visible and that they were to report only colors of which they felt certain. Inspection of the test gratings lasted as long as the subjects wished. Then, while standing at their original positions, they filled in answers to the following questions, which were on typed forms: (1) "What, if any, colour can you see on the narrow stripes?" (2) "What, if any, colour can you see on the wide stripes?" (3) "What, if any, colour can you see where the narrow and wide stripes overlap?" (4) "Can you in any way control the colour in the overlap region, perhaps by concentrating on the narrow or wide stripes, or by scanning from the top or bottom towards the middle of the pattern?" (5) "What, if any, is the strongest colour you see on the pattern?"

The results of all three studies were very similar and can be briefly described. Nineteen-exactly half-of the subjects reported two-color aftereffects, one for narrow and one for wide stripes. Of these, 13 saw the stronger of the two colors in the overlap region, 2 saw the weaker, and 4 reported no color there. There was no consistent relationship of color strength to stripe width. Among the remaining 19 subjects, 5 saw no color aftereffects anywhere on either test pattern, and 14 saw only a single color aftereffect. This was not systematically related to wider or narrower stripes, and 7 subjects reported that the single color was present in the overlap region. No obvious differences were found between patterns such as those shown in Figure 1 and Figure 2. Most significantly of all, no subject reported any ability to control the color seen in the overlap region.

In summary, these studies provided no evidence to suggest that subjects can attend selectively to the outputs of separate frequency channels that are differentially responsive to the two frequencies contained in the overlap region of the composite gratings. The subjects could not control the color seen in the overlap. There was some tendency for this region to take on the color of the strongest aftereffect visible to the subject, but this tendency was not universally reported.

It is worth noting that these reports closely resemble the experiences of the author during several intensive tests. With two aftereffect colors, the overlap region always took the color of the stronger. There was no conscious control of color in the overlap, which could only be changed by further inspecting the appropriate adaptation grating until the weaker aftereffect was suitably topped up.

What is to be made of these results? They certainly militate against any simple attempt to equate frequency analysis with attentional mechanisms, and therefore against the proposal of Julesz (1980). Under the conditions studied, it was not possible to demonstrate volitional selectivity to different spatial frequencies.

Now, it might be argued that contingent color aftereffects are not a suitable tool for probing attentional capabilities. By their nature, it could be said, aftereffects are preattentive or automatic (Neisser, 1967), so that it is to be expected that subjects are unable to influence the color seen on particular areas of a test pattern. Indeed, this result is much of a piece with the failure to make color aftereffects contingent upon more cognitive stimulus features, as informally reported by a number of investigators (Stromeyer, 1978). This objection may well be valid, but it is of no help to those who see a connection between attention and frequency analysis, because since the color aftereffects employed in these studies were contingent upon spatial frequency, rather than upon any factor such as stripe width (Harris, 1970; Stromeyer, 1978), their appearance presupposes a prior frequency analysis. That is, if the aftereffects are preattentive, so must be the frequency analysis upon which they are contingent, in which case the frequency analysis cannot be a mechanism of attention and cannot be the basis of perceptual zooming.

If the above argument is accepted, then it not only leads to a rejection of the Julesz proposal but also bears on the hypothesis outlined by Levinson and Frome (1979), which was apparently endorsed by Julesz and Schumer (1981). The idea in this case was that attentional focusing is distinct from, and in fact precedes, spatial frequency analysis, allowing window (and possibly other) functions to be imposed on the system prior to frequency analysis. However, if this were so, it might be difficult to explain even the occurrence of the usual frequency-contingent color aftereffects. Certainly the failure of selective attention in the present studies would become hard to understand, because if frequency analysis can be restricted to a particular portion of the visual field, it should be possible with a composite grating to at- 
tend to the thin stripes between any two thick stripes and to see only the color associated with the thin stripes. However, the subjects were just not able to do that. They could not discount part of the field and thereby select what color to perceive. The color of the overlap region was determined by the relative strengths of the aftereffects and by the antagonism between them. The aftereffects themselves appear to arise from a stage of visual processing prior to that at which conscious attention becomes effective but subsequent to frequency analysis.

In conclusion, the studies reported here provide no support for the idea that what spatial frequency analysis can buy for the visual system is attentional capability.

\section{REFERENCES}

Blakemore, C., \& Sutron, P. Size adaptation: A new aftereffect. Science, 1969, 166, 245-247.

Campbelt, F. W., \& Robson, J. G. Application of Fourier analysis to the visibility of gratings. Journal of Physiology, 1968, 197, 551-566.
Frome, F. S., Levinson, J. Z., Danielson, J. T., \& Clavadetscher, J. E. Shifts in perception of size after adaptation to gratings. Science, 1979, 206, 1327-1329.

HARris, C. S. Effect of viewing distance on a color aftereffect specific to spatial frequency. Psychonomic Science, 1970, 21, 350. (Abstract)

JuLEsz, B. Spatial frequency channels in one-, two-, and threedimensional vision: Variations on a theme by Békésy. In C. S. Harris (Ed.), Visual coding and adaptability. Hillside, N.J: Erlbaum, 1980.

Julesz, B., \& Schumer, R. A. Early visual perception. Annual Review of Psychology, 1981, 32, 575-627.

Levinson, J. Z., \& Frome, F. S. Perception of size of one object among many. Science, 1979, 206, 1425-1426.

Lovegrove, W. J., \& Oven, R. Color adaptation of spatial frequency detectors in the human visual system. Science, 1972, 176, 541-543.

Neisser, U. Cognitive psychology. New York: Appleton, 1967.

Stromeyer, C. F. Form-color aftereffects in human vision. In R. Held, H. Leibowitz, \& H.-L. Teuber (Eds.), Handbook of sensory physiology (Vol. 8). New York: Springer-Verlag, 1978.

(Manuscript received February 23, 1983; accepted for publication March 7, 1983.) 